DOI $10.35381 / \mathrm{cm} \cdot v 6 \mathrm{i} 10.205$

\title{
Emprendimiento desde la práctica del reciclaje ecológico
}

\section{Entrepreneurship from the practice of ecological recycling}

\author{
Alexandra Jacqueline Loor Moreira \\ Jackyloor2012@hotmail.com \\ Universidad Laica Eloy Alfaro de Manabí \\ Ecuador \\ https://orcid.org/0000-0002-5942-6212 \\ Claudia Analia Molina Quiroz \\ anamolinaq@gmail.com \\ Universidad Laica Eloy Alfaro de Manabí \\ Ecuador \\ https://orcid.org/0000-0001-7396-3519 \\ Aarón Leonel Baduy Molina \\ Aleon badmol@hotmail.com \\ Universidad Laica Eloy Alfaro de Manabí \\ Ecuador \\ https://orcid.org/0000-0003-3081-8359
}

Recibido: 20 de julio de 2019 Aprobado: 15 de agosto de 2019

\section{RESUMEN}

La investigación tuvo por objetivo analizar el emprendimiento desde la práctica del reciclaje ecológico en las instituciones educativas de Manta, Ecuador. Metodológicamente se fundamentó en una tipología descriptiva. Desde una educación creativa e innovadora, pueden desarrollarse en los estudiantes para configurarse como emprendedores, de ese modo, la escuela se constituye en un factor primordial para el crecimiento humano, se puede generar la innovación y creatividad al impulsar proyectos para el desarrollo de ideas de emprendimiento ecológico que además permitan formar en valores, entre estas actividades se encontrarían: servicio de reciclaje a domicilio, asesoramiento ecológico para instituciones públicas y privadas, diseño y 
acondicionamiento de áreas verdes, diseño de app con visión ecológica, podrían ser algunos de los emprendimientos a generar.

Descriptores: Ecología humana; Ética ambiental; Empresario; Trabajador independiente.

\section{ABSTRACT}

The research aimed to analyze entrepreneurship from the practice of ecological recycling in educational institutions in Manta, Ecuador. Methodologically it was based on a descriptive typology. From a creative and innovative education, they can be developed in the students to configure themselves as entrepreneurs, in this way, the school is a fundamental factor for human growth, innovation and creativity can be generated by promoting projects for the development of ideas of ecological entrepreneurship that also allow training in values, among these activities would be: home recycling service, ecological advice for public and private institutions, design and conditioning of green areas, app design with ecological vision, could be some of the ventures to generate.

Descriptores: Human ecology; Environmental ethics; Entrepreneurs; Self employed.

\section{INTRODUCCIÓN}

La educación transita hacia una transformación que coadyuve a estar en consonancia con los retos globales, de allí que existan instituciones educativas que dan adoptado el enfoque de emprendimiento como un valor agregado para formar a los estudiantes en proporcionalidad de asumir un aprendizaje para la vida que por medio de competencias contribuya a formalizar un ciudadano en posibilidad de contribuir efectivamente con el progreso de la sociedad. En este sentido, la actual investigación indaga sobre el emprendimiento escolar como una posibilidad de germinar ideas innovadoras por parte de los estudiantes, lo cual permitirá tener conciencia de la importancia de ser proactivo, independiente, para afrontar con prestancia su crecimiento integral.

Es así que se toma la perspectiva del emprendimiento como una acción no enriquecedora para la edad escolar desde el punto de vista monetario, sino como un enfoque formativo, aunado a generar una conciencia ecológica en conformidad de propiciar mediante el emprendimiento ecológico, una dualidad que contribuya a un aprendizaje significativo para el estudiante, siendo pertinente contar con programas formativos para que las 
instituciones educativas, propicien el enfoque de emprendiendo escolar ecológico. Aldana Zavala \& Colina Ysea (2019), plantean que:

Es necesario educar con una visión de ciudadanía planetaria, esto implica redescubrir la esencia de la educación, siendo esta educar y formar para la humanidad, para la vida, siendo indispensable transcender el conocimiento fragmentado, mecanicista, por un aprendizaje fomentado desde la investigación, lo cual permitirá contar con investigadores críticos, reflexivos, éticos, que comprendan que el conocimiento se actualiza en la medida que se obtienen nuevos resultados investigativos (p. 158).

Así se puede promover un currículo flexible, dinámico, integrador de visiones disciplinarias para tramitar en la consecución de un estudiante en capacidad de ser crítico, reflexivo, investigador, desde el aporte generado al contexto ecológico, situación que lo constituye en un ciudadano global, esto debe ser complementado en ser emprendedor, por cuanto el modelo asalariado se encuentra en crisis, originando múltiples problemas de índole económico y social, siendo el emprendimiento una alternativa viable para contrarrestar la pobreza generada del desempleo, Schumpeter, J., (1942), define al emprendedor como "persona dinámica y fuera de lo común, que promueve nuevas combinaciones o innovaciones".

Tales competencias desde una educación creativa e innovadora, pueden desarrollarse en los estudiantes para configurarse como emprendedores, de ese modo, la escuela se constituye en un factor primordial para el crecimiento humano, así se diferenciarán las instituciones educativas emprendedoras de las no emprendedoras, siendo esto un agregado que los padres y representantes tendrán en cuenta a la hora de seleccionar la institución para que sus hijos se formen. Aldana, Salón \& Guzmán (2019), complementan al indicar que:

Entra en juego la divergencia del aprendizaje, ante lo cual, no puede enseñarse de modo lineal, por cuanto unos individuos tendrán potencialidades para aprender hacer unas cosas y otras no. De allí que no puede hablarse de negatividades, sino de potencialidad en la persona ( $p$. $65)$. 
Una educación basada en el emprendimiento ecológico, promueve las potencialidades de los estudiantes para posicionarse en ventaja en comparación con los estudiantes no emprendedores, por cuanto unos tendrán una personalidad generadora de cambios, mientras que la otra posiblemente asuma posturas pasivas frente a las adversidades sociales. Hay que tener en cuenta que en el Ecuador se generan alrededor de 11.341 toneladas diarias de residuo, es decir, un aproximado de 4'139.512 Tm/año, de los cuales $61,4 \%$ son orgánicos, papel + cartón $9.4 \%$, plástico $11 \%$, vidrio $2.6 \%$, chatarra $2.2 \%$, y otros 13.3\% (Ministerio del Ambiente, 2014).

Esta visión permite analizar el emprendimiento desde la práctica del reciclaje ecológico en las instituciones educativas de Manta, Ecuador, posibilitándose la puesta en práctica de un currículo flexible, contextualizado a la realidad social local, contribuyéndose a promover la formación de los actores educativos en aras de sensibilizar al fomento del emprendimiento en las escuelas.

\section{REFERENTES TEÓRICOS}

El emprendimiento escolar es un enfoque que permite generar potencialidades en los estudiantes, Paternina De La Ossa (2018), destaca que "Las actitudes emprendedoras que los jóvenes van desarrollando les permite visionar su futuro, proyectar su norte, es decir saber que quieren y hacia donde construyen sus metas, identifican oportunidades en su entorno y construyen sus sueños basados en realidades" (p. 6), de ese modo, se constituye en un proyecto de vida que además contribuye a la formación bioética de la persona en razón de estar en concordancia con la preservación ecológica y proyección de contribuir a un mundo mejor. Por otro lado, la Comisión Nacional de la Micro y Pequeña Empresa (CONAMYPE), quien plantea que el:

Emprendimiento es una manera de pensar y actuar orientada hacia la creación de riqueza para aprovechar las oportunidades presentes en el entorno o para satisfacer las necesidades de ingresos personales generando valor a la economía y a la sociedad. Mientras que el Emprendedor: Es una persona con capacidad de innovar; entendida esta como la capacidad de generar bienes y 
servicios de una forma creativa, metódica, ética, responsable y efectiva. (CONAMYPE, 2014, p. 13).

El emprendimiento puede ser moldeado a las diversas perspectivas o necesidades, en este caso al ámbito escolar - ecológico, en complemento, la (CONAMYPE, 2014, p. 13), plantea que el emprendimiento se clasifica en:

1. Emprendimiento por Necesidad: Acción empresarial iniciada por personas que al momento de tomar la decisión de poner en marcha una actividad económica lo hicieron motivados por la falta de ingresos necesarios para su subsistencia (o por el deseo de obtener una fuente de ingreso adicional). Esta categoría se divide en dos tipos: emprendimiento de subsistencia y emprendimiento tradicional, las cuales se presentan a continuación:

1.1. Emprendimiento de Subsistencia: Acción empresarial dirigidas a generar ingresos diarios para vivir (autoempleo), sin una planificación o sin una visión de crecimiento y que tiende a no generar excedentes.

1.2. Emprendimiento Tradicional: Acción empresarial dirigida a la generación de ingresos que cuentan con una estructura organizacional y que utilizan el conocimiento técnico para la generación de excedentes que permiten la acumulación. Tienden a desarrollar su actividad en la formalidad, en mercados y sectores tradicionales de la economía sin elementos diferenciadores en sus productos y servicios.

2. Emprendimiento por Oportunidad: Acción empresarial iniciada por personas que al momento de tomar la decisión de poner en marcha una actividad económica lo hicieron motivados por la identificación de una oportunidad de mercado. Esta categoría se divide en dos tipos:

2.1. Emprendimiento Dinámico: Acción empresarial con alto potencial de crecimiento donde el uso del conocimiento, la gestión tecnológica y del talento humano, el potencial de acceso a recursos de financiación/inversión y una 
estructura de gobierno corporativo les permite generar una ventaja competitiva y diferenciación en sus productos o servicios.

2.2. Emprendimiento de Alto Impacto: Empresas con capacidad para transformar y dinamizar las economías a través de procesos sistemáticos de innovación y generación de empleo. Es una empresa que crece rápida y sostenidamente, ya que cuenta con altos niveles de financiación o de inversionistas.

Son diversos los tipos de emprendimiento, así como los enfoques del mismo, teniendo en consideración el Ecosistema de emprendimiento,

Es la comunidad de negocios, apoyada por un con texto público de leyes y prácticas de negocios formada por una base de organizaciones y personas interactuantes que producen y asocian ideas de negocios, habilidades, recursos financieros y no financieros que resultan en empresas dinámicas (CONAMYPE, 2014, p. 15).

El enfoque permite que las organizaciones funciones como incubadoras de emprendimiento, siendo esta una posibilidad para las instituciones educativas, las cuales podrían en futuro, generar el apoyo a emprendimientos de las familias, Nuñez Torrón, (2017) complementa diciendo que "por ecosistema emprendedor, se entiende todo aquel contexto y entorno que facilitan el surgimiento de empresas y proyectos empresariales" (p. 1), de ese modo, podrá existir la posibilidad de proyectar la consecución de una institución educativa conformada para asumir pedagógicamente los retos globales en prosecución de un ciudadano acorde a favorecer el crecimiento integral de la sociedad.

\section{METODOLOGÍA DE LA INVESTIGACIÓN}

La metodología fue una investigación tipo descriptiva, Arias (2009), la describe como la investigación que "consiste en la caracterización de un hecho, fenómeno o supo con establecer su estructura o comportamiento" (p. 64), apoyada en un diseño no experimental, por cuanto no se manipularon deliberadamente las variables de estudio, sino que se realizó una descripción de los datos obtenidos. Al respecto Hernández, Fernández y Baptista (2008), consideran que en los estudios no experimentales "lo que 
hacemos es observar fenómenos tal y como se dan en su contexto natural para después analizarlos" (p. 208).

La población de acuerdo a Tamayo y Tamayo (2007), "Es la totalidad del fenómeno a estudiar donde las unidades de población poseen una característica común la cual se estudia y da origen a los datos de la investigación" (p. 114), la cantidad de población estuvo conformada por 46 estudiantes de instituciones educativas de Manta, Ecuador a quienes se les aplicó una encuesta, mediante un instrumento tipo escalamiento de Likert de cinco alternativas de respuestas, aplicándose el coeficiente de Alfa de Cronbach, obteniéndose un resultado de 0,92 , lo cual cataloga al instrumento en un rango de muy alta confiabilidad.

\section{RESULTADOS}

Los resultados se plantean desde la perspectiva generada por los estudiantes que participaron en la investigación de ese modo, se cumplió con el objetivo de analizar el emprendimiento desde la práctica del reciclaje ecológico en las instituciones educativas de Manta, Ecuador, para lo cual se presentan los siguientes resultados:

\section{Promoción del emprendimiento escolar Gráfico 1}

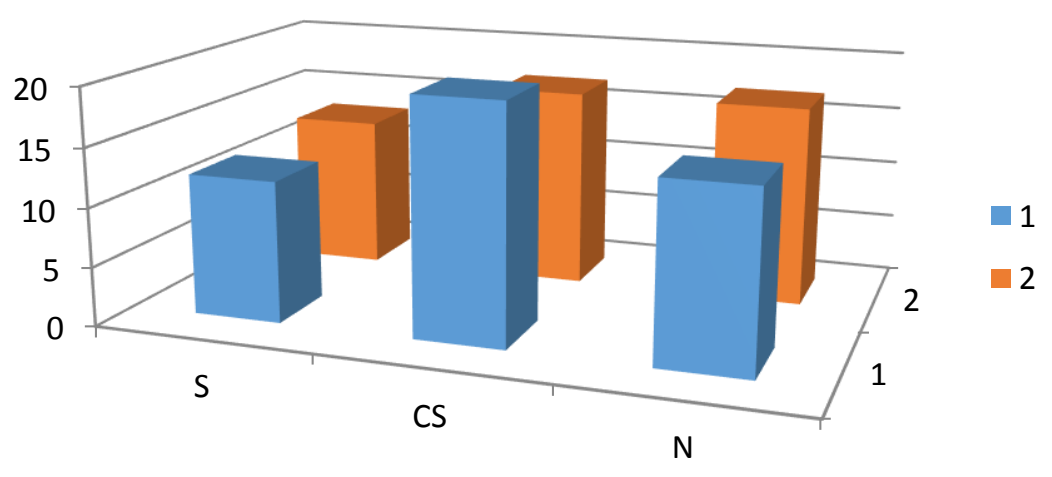


Con base en los datos estadísticos obtenidos de la aplicación de la encuesta, se tiene que para el ítems 1, la opción siempre tuvo una representación estadística del 25\%, así mismo la opción casi siempre un $43 \%$, y alternativa nunca el $32 \%$ Por otro lado, para el ítems 2, la opción siempre tuvo una representación estadística del 28\%, así mismo la opción casi siempre un $36 \%$, y alternativa nunca el $36 \%$

\section{Formación ecológica \\ Gráfico 2}

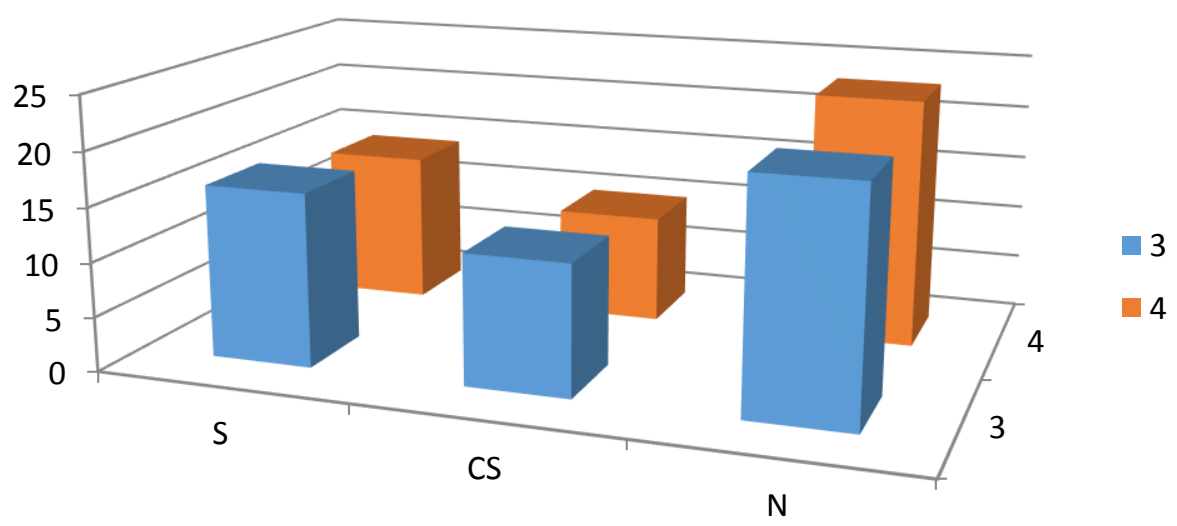

En relación a los datos estadísticos obtenidos de la aplicación de la encuesta, se tiene que para el ítems 2, la opción siempre tuvo una representación estadística del 33\%, así mismo la opción casi siempre un $24 \%$, y alternativa nunca el $43 \%$

Por otro lado, para el ítems 3, la opción siempre tuvo una representación estadística del $30 \%$, así mismo la opción casi siempre un $21 \%$, y alternativa nunca el $49 \%$ 
Año VI. Vol. VI. No10. Enero - Julio 2020

Hecho el depósito de ley: pp201602FA4721

ISSN-L: 2542-3029; ISSN: 2610-802X

Universidad Nacional Experimental Francisco de Miranda (UNEFM). Santa Ana de Coro. Venezuela

Alexandra Jacqueline Loor Moreira; Claudia Analia Molina Quiroz; Aarón Leonel Baduy Molina

\section{Actividades de reciclaje}

\section{Gráfico 3}

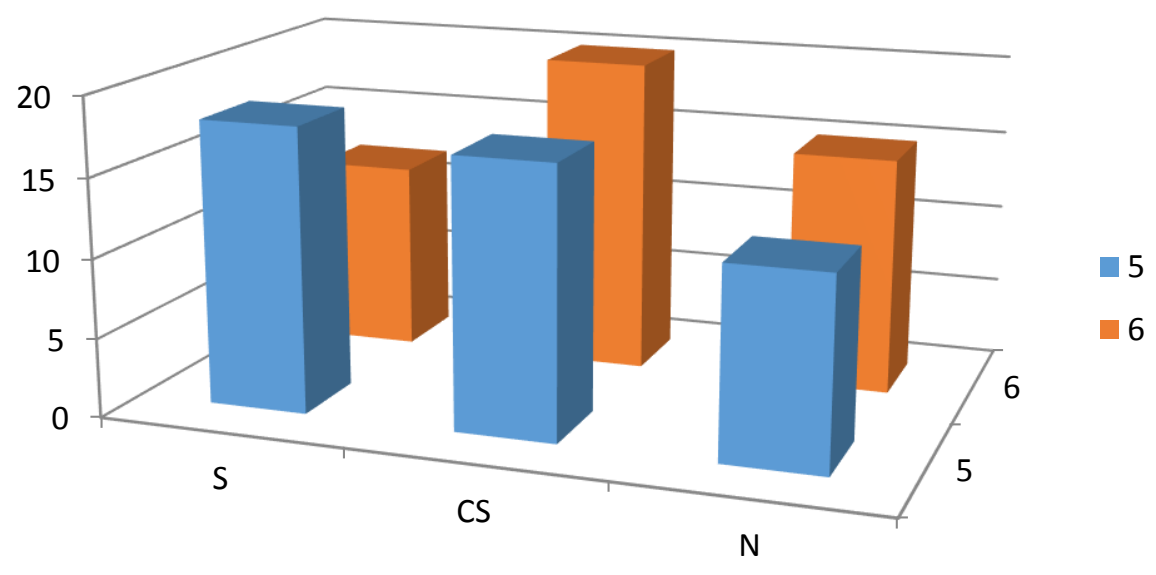

Con base en los datos estadísticos obtenidos de la aplicación de la encuesta, se tiene que para el ítems 5, la opción siempre tuvo una representación estadística del 38\%, así mismo la opción casi siempre un $36 \%$, y alternativa nunca el $26 \%$

Por otro lado, para el ítems 6 , la opción siempre tuvo una representación estadística del $25 \%$, así mismo la opción casi siempre un $43 \%$, y alternativa nunca el $32 \%$

\section{Apoyo familiar}

\section{Gráfico 4}

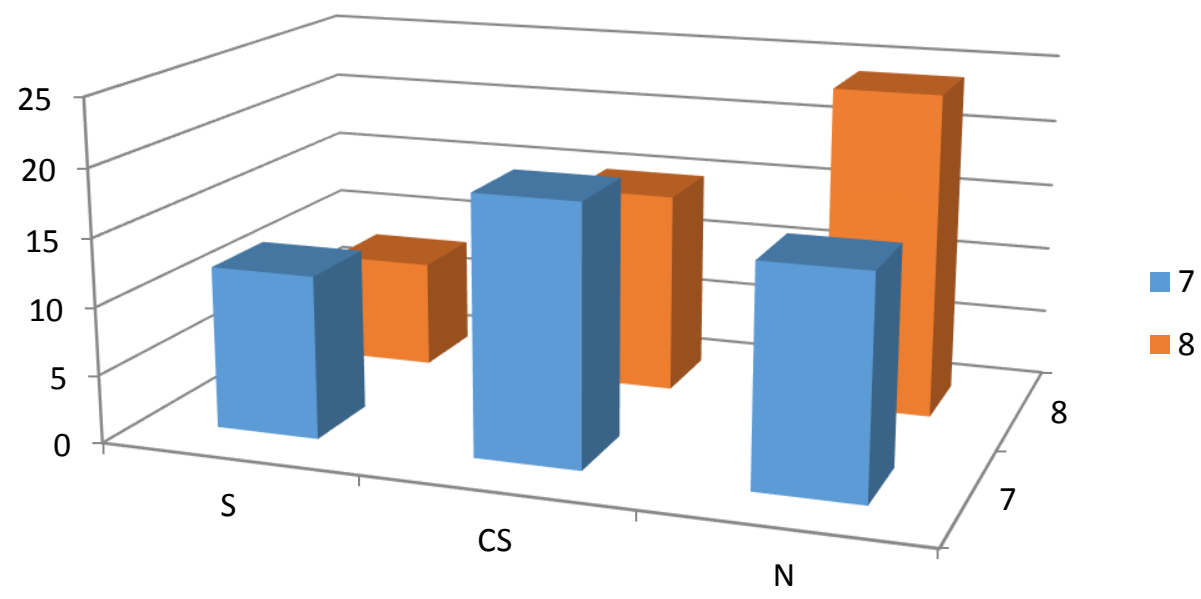


En función de los datos estadísticos obtenidos de la aplicación de la encuesta, se tiene que para el ítems 7, la opción siempre tuvo una representación estadística del 26\%, así mismo la opción casi siempre un $40 \%$, y alternativa nunca el $34 \%$

Por otro lado, para el ítems 8, la opción siempre tuvo una representación estadística del $17 \%$, así mismo la opción casi siempre un $32 \%$, y alternativa nunca el $51 \%$

\section{DISCUSIÓN}

Los resultados evidencian que no se ha generado el emprendimiento ecológico en las instituciones educativas de modo efectivo, lo cual no permite fomentar la responsabilidad social empresarial como un valor fundamental en la formación de los estudiantes, siendo necesario implementar acciones que permitan emprender desde una concepción ecológica, Arias \& Sarmiento (2017). Por lo tanto, el instituto de emprendimiento avanzado (2018), plantea que:

Muchos emprendedores se lanzan a crear una nueva actividad sin tener la educación financiera adecuada. No son conscientes de su importancia y creen que irán aprendiendo sobre la marcha, pero luego detalles como las fuentes de financiación, la planificación financiera o la gestión de los créditos los desborda.

Siendo importante en la escuela a la hora de promover emprendimientos ecológicos, focalizar la educación financiera como generador de una visión para el manejo sostenible de los recursos financieros, siendo esta una posibilidad para también sensibilizar sobre el ahorro como alternativa para el crecimiento familiar.

\section{CONCLUSIONES}

El emprendimiento ecológico escolar, posibilita la integración de las familias a los proyectos educativos de los estudiantes, haciendo posible que éstos se formen con una visión amplia y proactiva para asumir los diversos retos sociales, preparándose además para ser líderes transformadores en capacidad de promover un ambiente de mayor armonía para la coexistencia humana con los seres vivos del planeta. 
Así mismo se puede generar la innovación y creatividad al impulsar proyectos para el desarrollo de ideas de emprendimiento ecológico que además permitan formar en valores, entre estas actividades se encontrarían: servicio de reciclaje a domicilio, asesoramiento ecológico para instituciones públicas y privadas, diseño y acondicionamiento de áreas verdes, diseño de app con visión ecológica, podrían ser algunos de los emprendimientos a generar.

Esto a su vez, podría ayudar a los estudiantes a formarse en competencias transversales que les ayuden a descubrir sus posibles profesiones, entre las cuales podrían estar arquitectos, ingenieros, consultores gerenciales, trabajar en el área de informática, así como tener la base para emprender negocios sustentables, siendo así que la educación forma desde un contexto complejo y holístico.

\section{REFERENCIAS CONSULTADAS}

1. Aldana Zavala, J., \& Colina Ysea, F. (2019). Marketing verde en la conformación de una ciudadanía planetaria en el ámbito educativo latinoamericano. Revista San Gregorio, O(31), 150-161. doi:http://dx.doi.org/10.36097/rsan.v0i31.972

2. Aldana, J., Salón, M., \& Guzmán, N. (2019). Liderazgo sistémico en las competencias gerenciales docentes universitarias. CIENCIAMATRIA, 5(8), 50-74. Recuperado a partir http://cienciamatriarevista.org.ve/index.php/cm/article/view/87

3. Arias, E., \& Sarmiento, D. (2017). RESPONSABILIDAD SOCIAL EMPRESARIAL EN FALCÓN. UN ANÁLISIS DE CONTENIDO. Revista Arbitrada Interdisciplinaria Koinonía, 1(1), 22-42. Recuperado de http://fundacionkoinonia.com.ve/ojs/index.php/revistakoinonia/article/view/13/6

4. Arias, F. (2009). El Proyecto de Investigación. Guía para su elaboración. Caracas: Epísteme. Quinta Edición.

5. CONAMYPE (2014). Política nacional de emprendimiento. Recuperado de http://www.conamype.gob.sv/wp-content/uploads/2014/08/Poli\%CC\%81tica-deEmprendimiento.pdf.

6. Hernández, Fernández y Baptista (2008). Metodología de la investigación. México, Mc Graw Hill Hispanoamericana. Hill Internacional 
7. Instituto de emprendimiento avanzado (2018). Educación financiera para emprendedores. ¿Qué es y cuáles son sus beneficios? Recuperado de https://ieavanzado.com/blog/formacion/educacion-financiera-paraemprendedores

8. Nuñez Torreón, A. (2017). Que son y en qué consisten los ecosistemas emprendedores. Recuperado de http://www.ticbeat.com/empresa-b2b/que-son-yen-que-consisten-los-ecosistemas-emprendedores/

9. Paternina De La Ossa (2018). El emprendimiento escolar, una estrategia de identificación de talentos excepcionales. Recuperado de http://www.revistaespacios.com/a18v39n49/a18v39n49p05.pdf

10. República del Ecuador. Ministerio del Ambiente. (2014). Informe de logros alcanzados por el programa nacional de gestión integral de desechos sólidos. Recuperado de

HYPERLINK "http://suia.ambiente.gob.ec/documents/10179/254996/Informe+Gestion+resumid o+MAE-PGNIDS+2010-2013.pdf/95b81b2d-b2a1-4a98-b7a3-22920795e78a" http://suia.ambiente.gob.ec/documents/10179/254996/Informe+Gestion+resumid o+MAE-PGNIDS+2010-2013.pdf/95b81b2d-b2a1-4a98-b7a3-22920795e78a

11. Tamayo y Tamayo (2009). El proceso de investigación científica. Limusa. México.

\section{REFERENCES CONSULTED}

1. Aldana Zavala, J., \& Colina Ysea, F. (2019). Green marketing in the conformation of a planetary citizenship in the Latin American educational field. San Gregorio Magazine, 0 (31), 150-161. doi: http: //dx.doi.org/10.36097/rsan.v0i31.972

2. Aldana, J., Salón, M., \& Guzmán, N. (2019). Systemic leadership in university teaching management skills. SCIENCE, 5 (8), 50-74. Recovered from http://cienciamatriarevista.org.ve/index.php/cm/article/view/87

3. Arias, E., \& Sarmiento, D. (2017). CORPORATE SOCIAL RESPONSIBILITY IN FALCÓN. AN ANALYSIS OF CONTENT. Interdisciplinary Arbitrated Review $\begin{array}{lllll}\text { Koinonía, } & 1 & \text { (1), } 22-42 . & \text { Recovered from }\end{array}$ http://fundacionkoinonia.com.ve/ojs/index.php/revistakoinonia/article/view/13/6

4. Arias, F. (2009). The Research Project Guide for its elaboration. Caracas: Epistle me. Fifth edition. 
5. CONAMYPE (2014). National entrepreneurship policy. Recovered from http://www.conamype.gob.sv/wp-content/uploads/2014/08/Poli\%CC\%81tica-deEmprendimiento.pdf.

6. Hernández, Fernández and Baptista (2008). Investigation methodology. Mexico, Mc Graw Hill Hispanic American. Hill International

7. Advanced Entrepreneurship Institute (2018). Financial education for entrepreneurs. What is it and what are its benefits? Recovered from https://ieavanzado.com/blog/formacion/educacion-financiera-paraemprendedores

8. Nuñez Torreón, A. (2017). What are and what entrepreneurial ecosystems consist of. Recovered from http://www.ticbeat.com/empresa-b2b/que-son-y-en-queconsisten-los-ecosistemas-emprendedores/

9. Paternina De La Ossa (2018). School entrepreneurship, a strategy for identifying exceptional talents. Recovered http://www.revistaespacios.com/a18v39n49/a18v39n49p05.pdf

10. Republic of Ecuador. Ministry of Environment (2014). Report of achievements achieved by the national program of integral solid waste management. Recovered from HYPERLINK "http://suia.ambiente.gob.ec/documents/10179/254996/Informe+Gestion+resumid o+MAE-PGNIDS+2010-2013.pdf/95b81b2d-b2a1-4a98-b7a3-22920795e78a" http:

//suia.ambiente.gob.ec/documents/10179/254996/Informe+Gestion+resumido+M AE-PGNIDS+2010-2013.pdf/95b81b2d-b2a1-4a98-b7a3-22920795e78a

11. Tamayo and Tamayo (2009). The process of scientific research. Limusa Mexico. 